\title{
Dynamic changes in phosphoinositide levels control ion channel activity
}

\author{
Diomedes E. Logothetis • Bernd Nilius
}

Received: 19 June 2007 / Accepted: 20 June 2007 /Published online: 20 July 2007

(C) Springer-Verlag 2007

In the last decade, following the initial reports by Hilgemann and Ball (Science 273:956-959, 1996) and Fan and Makielski (J. Biol. Chem. 272:5388-395, 1997), dynamic changes in the levels of plasma membrane phosphoinositides have been shown to control the activity of most ion channels and transporters. This form of regulation of the activity of membrane proteins complemented the well-studied phosphoinositide control of the localization of cytosolic proteins, such as in vesicular trafficking, cytoskeletal remodeling, recruitment to the plasma or to the nuclear membranes. In this special issue, we first examine phosphorylation and dephosphorylation of phosphoinositides, as well as functional consequences of such modifications, the atomic level interactions of phosphoinositides with a number of different proteins, the evidence for local $\mathrm{PIP}_{2}$ signals, and the dynamic way in which changes in plasma membrane phosphoinositide levels can be visualized by introducing novel approaches. We next turn to phosphoinositide regulation of the activity of a number of different ion channels, such as potassium inward rectifiers (Kir), a two-pore domain potassium $\left(\mathrm{K}_{2 \mathrm{P}}\right)$ leak, the voltage-gated human ether-a-go-go related gene (HERG) and muscarinic (M)-type potassium, the hyperpolarization-activated cyclic

\footnotetext{
D. E. Logothetis $(\bowtie)$

Department of Structural and Chemical Biology,

Mount Sinai School of Medicine of New York University,

New York, NY 10029, USA

e-mail: diomedes.logothetis@mssm.edu

B. Nilius

Division Physiology, Department of Molecular Cell Biology, Campus Gasthuisberg, Catholic University of Leuven,

Herestraat 49, bus 802 ,

3000 Leuven, Belgium
}

nucleotide-gated ( $\mathrm{HCN})$, the voltage-gated $\mathrm{Ca}^{2+}$, TRP, the epithelial sodium $(\mathrm{ENaC})$, and purinergic $\mathrm{P} 2 \mathrm{X}$ receptors.

Yuntao Mao and Helen Yin review the evidence showing that the phosphatidylinositol 4-phosphate 5 kinases (PIP5Ks), which make PIP(4,5) $\mathrm{P}_{2}$ (or $\mathrm{PIP}_{2}$ ) from PI(4)P, show spatial and temporal regulation and isoform-specific functions, such as actin polymerization, receptor-mediated endocytosis, focal adhesion dynamics, synaptic vesicle physiology, membrane ruffling, phagocytosis, etc.

Dora Visnjic and Hrvoje Banfic review recent studies of nuclear phosphoinositide signaling, which has been documented in the nuclei of many different cell types. They focus on phosphatidylinositol-specific phospholipase C (PIPLC) and phosphoinositide 3-kinase (PI3K) by examining their subnuclear localization, mechanisms of activation, and possible physiological roles in the regulation of cell cycle, survival, and differentiation.

Daniel Biero and colleagues in Christophe Erneux's laboratory review the different phosphoinositide phosphatases and illustrate the importance of these enzymes in cell regulation by their involvement in several human diseases such as Lowe syndrome, X-linked myotubular myopathy, cancer, diabetes, and bacterial infection.

Avia Rosenhouse-Dantsker and Diomedes Logothetis review 25 crystallographic complexes of phosphoinositides with different proteins and summarize molecular features that characterize the binding, such as the types and numbers of residues that bind phosphoinositides, the determinants of binding affinity and specificity, and the protein conformational changes induced by phosphoinositide binding. They also consider implications that these generalizations may have for phosphoinositide binding to ion channels, where no structures of complexes have become available yet.

Don Hilgemann reviews cases in which $\mathrm{PIP}_{2}$ is proposed to function in a localized way, and he considers different 
mechanistic models to account for local $\mathrm{PIP}_{2}$ action. Such considerations require restrictions in $\mathrm{PIP}_{2}$ diffusion and a number of mechanisms to achieve this are discussed, such as the action of the cytoskeleton, the binding to interacting proteins and specialized membrane domains. It is speculated that molecular $\mathrm{PIP}_{2}$ signaling could take place in the absence of $\mathrm{PIP}_{2}$ gradients, and possibilities are discussed.

Peter Varnai and Tamas Balla review tools that have been developed, particularly through use of engineered protein domains, to visualize and manipulate phosphoinositide dynamics in live cells. They report on lipid-binding domains as tools to monitor phosphoinositide dynamics and discuss the different ways in which the localization of these protein domains to the membrane has been quantified. In manipulating phosphoinositide dynamics, they discuss how to target lipid-binding domains to different cellular compartments.

Diomedes Logothetis and colleagues review work on inwardly rectifying $\mathrm{K}^{+}$channels that aims to obtain molecular insights on how channel-PIP ${ }_{2}$ interactions control channel activity and gating. They map onto the threedimensional structure of Kir2.1 (also known as IRK1) the position of basic residues identified through site-directed mutagenesis to be involved in $\mathrm{PIP}_{2}$ sensitivity onto the three-dimensional structure of Kir2.1 (or IRK1). The localization of these putative PIP $_{2}$-interacting residues has given rise to a model of gating in which $\mathrm{PIP}_{2}$ is thought to act as the glue to tether the $\mathrm{N}$ and $\mathrm{C}$ termini of the channel to the membrane and exert a tangential force that pulls on the inner helices and accomplishes mechanical gating.

Jean Chemin and colleagues in the Honoré and Lazdunski laboratories present original work to study the regulation of the two-pore domain channel TREK-1 by $\mathrm{PIP}_{2}$ and other membrane phospholipids. They find that intracellular phospholipids inhibit basal channel activity and activation by membrane stretch, intracellular acidosis, and arachidonic acid. Poly-lysine treatment targeting endogenous anionic phospholipids reduces the inhibition and reveals channel stimulation by exogenous phosphoinositides. The distal C-terminal domain is implicated in this effect.

Jin-Song Bian and Thomas McDonald review the evidence for $\mathrm{PIP}_{2}$ interactions with the voltage-gated HERG channel, a rapidly activating delayed rectifier $\mathrm{K}^{+}$ current $\left(I_{\mathrm{kr}}\right)$, and its potential biological significance in the autonomic regulation of HERG channels that proceeds through the Gq-coupled receptor to change membrane $\mathrm{PIP}_{2}$ levels.

Simon Hughes and colleagues in the Tinker and Brown laboratories explore the mechanism of M-type $\mathrm{K}^{+}$current inhibition by the peptide bradykinin (BK) using original work. It was thought that $\mathrm{BK}$ inhibited $\mathrm{M}$ currents via an $\mathrm{IP}_{3}$-mediated release of $\mathrm{Ca}^{2+}$ from intracellular stores rather than by a depletion of $\mathrm{PIP}_{2}$ levels. Yet PLC $\delta-\mathrm{PH}-\mathrm{GFP}$ translocation from the membrane to the cytosol showed a similar pattern to that caused by a muscarinic agonist, which has been shown to inhibit $\mathrm{M}$ currents by $\mathrm{PIP}_{2}$ depletion. Hughes et al. utilized a better tool reporting on $\mathrm{PIP}_{2}$ levels, tubby-R332H-cYFP, which showed that BK produced about one third the translocation seen by the muscarinic agonist. This difference between the two agonists could be abolished when a BK-induced synthesis of $\mathrm{PIP}_{2}$ could be blocked.

Phillip Pian and colleagues in Steve Siegelbaum's laboratory explored through original work the modulation of HCN currents by signals that activated phospholipase C and, thus, hydrolyzed $\mathrm{PIP}_{2}$. $\mathrm{PIP}_{2}$ enhanced HCN activity by causing a rightward shift of voltage-dependent gating as was previously shown. Stimulation of receptors coupled to PLC had a similar effect on the voltage dependence of activation as $\mathrm{PIP}_{2}$. This paradoxical effect could be accounted for if, as in the study by Hughes and colleagues on the BK-mediated inhibition of $\mathrm{M}$ currents, $\mathrm{PIP}_{2}$ synthesis was blocked.

Ioannis Michailidis, Yun Zhang, and Jian Yang reviewed phosphoinositide regulation of high voltage-activated $\mathrm{Ca}^{2+}$ channels. Phosphoinositides have been shown to stabilize $\mathrm{Ca}^{2+}$ channel activity but also to produce a voltagedependent inhibition that can be antagonized by protein kinase A (PKA) phosphorylation. PIP $_{2}$ depletion and arachidonic acid together are responsible for the slow voltage-independent inhibition of $\mathrm{Ca}^{2+}$ currents seen with $\mathrm{Gq} / 11$-coupled receptor stimulation. A sufficient level of $\mathrm{PIP}_{2}$ seemed to also be necessary for $G \beta \gamma$-mediated inhibition. $\mathrm{PIP}_{3}$ promoted trafficking of $\mathrm{Ca}^{2+}$ channels to the plasma membrane.

Tibor Rohacs and Bernd Nilius review regulation of various TRP channels (e.g., TRPMs, TRPCs, TRPVs) in the context of $\mathrm{PIP}_{2}$ regulation of other ion channels. They discuss the possibility that $\mathrm{PIP}_{2}$ is a common regulator of mammalian TRP channels and focus on novel tools to study the diverse effects of $\mathrm{PIP}_{2}$ on these channels.

He-Ping Ma and colleagues in his and Douglas Eaton's laboratories explore regulation of $\mathrm{ENaC}$ by phosphoinositides. $\mathrm{PIP}_{2}$ amd $\mathrm{PIP}_{3}$ activate ENaC. Activation of PLC by purinergic $(\mathrm{P} 2 \mathrm{Y})$ receptor stimulation or EGF-induced activation reduce membrane $\mathrm{PIP}_{2}$ and consequently decrease channel activity. In contrast, stimulation of insulin-like growth factor-1 (IGF-1) receptor by aldosterone of insulin produces $\mathrm{PIP}_{3}$ and increases channel activity. Interactions of $\mathrm{PIP}_{2}$ and $\mathrm{PIP}_{3}$ with the $\mathrm{N}$ and $\mathrm{C}$ termini of distinct $\mathrm{ENaC}$ subunits of the channel are investigated. The implications of these agonist-induced effects to disease are discussed.

Qi Zhao, Diomedes Logothetis, and Philippe Seguela explored the regulation of purinergic $\mathrm{P} 2 \mathrm{X}$ receptors by phosphoinositides. Two receptors, $\mathrm{P} 2 \mathrm{X}_{2}$ and $\mathrm{P} 2 \mathrm{X}_{7}$, have 
previously been studied. Basic residues in the C-terminal region proximal to the membrane seem to be involved in interactions with $\mathrm{PIP}_{2}$ in both $\mathrm{P}_{2} \mathrm{X}_{2}$ and $\mathrm{P} 2 \mathrm{X}_{7}$ channels. Moreover, in original data from $\mathrm{P} 2 \mathrm{X}_{3}$ receptors found in DRG neurons, the authors showed that block of $\mathrm{PIP}_{2}$ levels inhibited $\mathrm{P} 2 \mathrm{X}_{3}$ currents.

Although the field of phosphoinositide regulation of membrane proteins has exploded in recent years, it is clear that we are still at the early stages of understanding the fundamental principles by which these important signaling phospholipids are able to control the activities of so many ion channels. To meet this challenge, novel tools that will enable us to directly monitor changes in phosphoinosites need to be developed. Because hot spots of putative $\mathrm{PIP}_{2}$-interacting residues are shared among very different channel types, it is likely that the mechanism by which specific channel-PIP ${ }_{2}$ interactions occur and how they lead to channel gating will be shared among different channel types. 